\title{
Exchange of a single amino acid residue in the cryptophyte phycobiliprotein lyase GtCPES expands its substrate specificity
}

\author{
Natascha Tomazic ${ }^{1}$, Kristina E. Overkamp ${ }^{1}$, Marco Aras ${ }^{1, \#, ~ A n t o n i o ~ J . ~ P i e r i k ~}{ }^{2}$, Eckhard \\ Hofmann $^{3}$, and Nicole Frankenberg-Dinkel ${ }^{1}$
}

\begin{abstract}
${ }^{1}$ Abteilung Mikrobiologie, Fachbereich Biologie, Technische Universität Kaiserlautern, Germany
${ }^{2}$ Abteilung Biochemie, Fachbereich Chemie, Technische Universität Kaiserslautern, Germany ${ }^{3}$ Proteinkristallographie, Fakultät für Biologie und Biotechnologie, Ruhr-Universität Bochum, Germany
\end{abstract}

Running title: Phycobiliprotein lyase substrate specificity

\# present address: Lehrstuhl Technische Biochemie, Technische Universität Dortmund, Germany

*To whom the correspondence should be addressed: Nicole Frankenberg-Dinkel, Abteilung Mikrobiologie, Fachbereich Biologie, Technische Universität Kaiserlautern, 67663 Kaiserslautern, Germany; nfranken@bio.uni-kl.de; Tel. +49 631205 2353; Fax: +49 6312053799.

Keywords: algae, light-harvesting complex (antenna complex), photosynthetic pigment, protein assembly, spectroscopy, structure-function

Phycobiliprotein lyase, phycobiliprotein, cryptophyte, phycoerythrobilin, Guillardia theta

\begin{abstract}
Cryptophyte algae are among the few eukaryotes that employ phycobiliproteins (PBP) for light harvesting during oxygenic photosynthesis. In contrast to the cyanobacterial PBP that are organized in large membrane-associated super complexes, the phycobilisomes, those from cryptophytes are soluble within the chloroplast thylakoid lumen. Their light-harvesting capacity is due to covalent linkage of several open-chain tetrapyrrole chromophores (phycobilins). Guillardia theta utilizes the PBP phycoerythrin PE545 with 15,16-dihydrobiliverdin (DHBV) in addition to phycoerythrobilin (PEB) as chromophores. Thus far, the assembly of cryptophyte PBPs is not yet completely understood but involves the action of PBP-lyases as shown for cyanobacterial PBP. PBP-lyases facilitate the attachment of the chromophore in the right configuration and stereochemistry. Here we present the functional characterization of eukaryotic S-type PBP lyase GtCPES from $G$. theta. We show GtCPES mediated transfer and covalent attachment of PEB to the conserved $\mathrm{Cys}^{82}$ of the acceptor PBP $\beta$-subunit $(\mathrm{PmCpeB})$ of Prochlorococcus marinus MED4. Based on the previously solved crystal structure, the GtCPES binding pocket was investigated using site-directed mutagenesis. Thereby, amino acid residues involved in phycobilin binding and transfer were identified. Interestingly, exchange of a single amino acid residue $\mathrm{Met}^{67}$ to Ala extended the substrate specificity to phycocyanobilin (PCB) likely by enlarging the substrate-binding pocket. Variant GtCPES_M67A binds both PEB and PCB forming a stable, colorful complex in vitro and in vivo produced in Escherichia coli. GtCPES_M67A is able to mediate PCB transfer to Cys ${ }^{82}$ of PmCpeB. Based on our data we postulate that a single amino acid residue determines the bilin-specificity of phycoerythrin S-type lyases but that additional factors regulate hand over to the target protein.
\end{abstract}


Phycobiliproteins (PBPs) are the photosynthetic light-harvesting structures of cyanobacteria, red algae and cryptophytes and are used to absorb regions of the visible light spectrum that are poorly covered by chlorophylls. Consequently, PBPs efficiently absorb light in the spectral region between 430 and 680 $\mathrm{nm}$ (1) They consist of apo-proteins with covalently linked open-chain tetrapyrrole molecules termed phycobilins. While PBPs in cyanobacteria and red algae are organized in large light-harvesting structures called phycobilisomes, those of cryptophyte algae are soluble in the thylakoid lumen of the chloroplast (2-4). Cryptophycean PBPs occur in large concentrations and are organized in $(\alpha \beta)\left(\alpha^{\prime} \beta\right)$ heterodimers (4). In contrast to cyanobacteria, cryptophytes only use a single type of PBP. The model species Guillardia theta utilizes phycoerythrin 545 (PE545) with an absorption maximum at $545 \mathrm{~nm}$ (5). It consists of the $\alpha$-subunit CPEA with bound 15,16-dihydrobiliverdin (DHBV) and the $\beta$-subunit CpeB loaded with three molecules of phycoerythrobilin (PEB) $(6,7)$. The $G$. theta nuclear genome encodes 16 CPEA copies that share high homology among each other but lack homologies to any other known protein (8). In contrast, the plastid-encoded $\beta$-subunit is highly similar to those of cyanobacteria and rhodophytes (2,9-12). Both subunits possess thioether-linked phycobilins at conserved cysteine residues $\left(\alpha-\right.$ Cys $^{19} ; \beta$-Cys ${ }^{82}, \beta$-Cys $\left.{ }^{155}\right)$, one is connected via a double linkage $\left(\beta-\mathrm{Cys}^{50 / 61}\right)(4)$. The biosynthesis of the open-chain tetrapyrrole chromophores is highly conserved among PBP containing organisms, and starts with cleavage of a heme molecule by heme oxygenase. The resulting product biliverdin IX $\alpha(\mathrm{BV})$ is the substrate of enzymes belonging to the ferredoxin-dependent bilin reductases and reduce the BV substrate at different positions $(13,14)$. G. theta was shown to possess a plastid encoded heme oxygenase and two nucleus encoded FDBRs, PEBA and PEBB involved in the conversion of BV to PEB via 15,16-DHBV (14). Following their synthesis, the phycobilins are posttranslationally attached to the PBP with the help of PBP-lyases, which mediate the efficient attachment to the specific cysteine residues of the apo-PBPs (15).

PBP-lyases are classified into E/F-, S/U- and T-type lyases, each being responsible to facilitate the attachment of a phycobilin to a specific cysteine residue (15) Within the genomes of G. theta we previously identified four different PBP-lyases: CPES, CPET, CPEX and CPEZ. All are encoded in the nuclear genome, except CPET, which was identified on the nucleomorph genome $(14,16)$. GtCPES from $G$. theta belongs to the S/U-type lyases and adopts a 10 -stranded $\beta$-barrel with a modified lipocalin fold (14). It specifically binds phycobilins with a reduced C15,C16- bond like 15, 16-DHBV and PEB (14). Binding of phycocyanobilin (PCB) with a rigid double bond between $\mathrm{C} 15$ and $\mathrm{C} 16$ was not observed. Based on a study of a CPES homolog from the cyanobacterium Prochlorococcus marinus MED4, GtCPES is hypothesized to be involved in the attachment of PEB to $\beta$-Cys ${ }^{82}$ (17). Likely, the lyase is not catalyzing the transfer reaction itself, but rather assists in the handover of the phycobilin in the correct configuration to allow stereospecific autocatalytic attachment.

In this study, we demonstrate the specific ability of GtCPES to support the transfer of PEB to Cys ${ }^{82}$ of CpeB from the cyanobacterium Prochlorococcus marinus MED4 (PmCpeB). Moreover, we identified individual amino acid residues involved in phycobilin binding and transfer with one single amino acid change expanding the substrate spectrum. Taken together our data emphasize that the narrow substrate specificity of the PE specific S-type PBP-lyases are due to a single amino acid residue.

\section{RESULTS}

GtCPES mediates transfer of PEB to $\mathrm{Cys}^{82}$ of PmCpeB. Although we have previously shown that GtCPES binds PEB and 15,16-DHBV with high affinity, an involvement in the chromophorylation of the $G$. theta PBP $\beta$-subunit at $\beta$-Cys ${ }^{82}$ has not yet been shown. This was mainly due to solubility issues of the recombinant $G$. theta CpeB apo-protein and we were not able to solve this issue until now (14). Furthermore, it is still unclear whether chromophorylation of PBP has to follow a specific order. In this regard, it has previously been reported that $\beta$-Cys ${ }^{155}$ is the first site of chromophorylation in phycocyanin (18) Therefore, we took another approach to investigate whether GtCPES is able to support PEB attachment to the PE $\beta$-subunit. We made use of $\beta$-PE (CpeB) from the cyanobacterium P. marinus MED4 (PmCpeB). This $\beta$-subunit is the only PBP in this organism and is highly degenerated, as it only possesses a single chromophorylation site at $\mathrm{Cys}^{82}(19,20)$. Recombinant $\mathrm{PmCpeB}$ can be purified in adequate amounts to test chromophore transfer employing GtCPES (17). To verify whether GtCPES is sufficient for correct bilin addition to $\mathrm{PmCpeB}$ we compared spontaneous and lyase mediated attachment of 3(Z)-PEB to PmCpeB by recording the fluorescence emission of assembled holo-CpeB. Covalent attachment was furthermore confirmed by zinc-blot analysis. PmCpeB lacking the cysteine 
attachment site (i.e. PmCpeB_C82A) served as a negative control (Figure 1). In all experiments, the lyase was used in excess to prevent the presence of free bilin in the sample. Spontaneous and lyasemediated attachment resulted in the formation of covalent fluorescent phycobiliprotein complexes (Figure 1AB). Reaction products (i.e. holo- $P m \mathrm{CpeB}$ ) were stronger and displayed a more stable fluorescence in presence of GtCPES with an emission maximum at $568 \mathrm{~nm}\left(\lambda_{\mathrm{ex}}=550 \mathrm{~nm}\right)$. Addition of free 3(Z)-PEB to apo-PmCpeB resembled spontaneous attachment as described before (17). These measurements are in agreement with data observed for the native Prochlorococcus lyase CpeS (17) and confirm the activity of GtCPES in mediating the correct attachment of PEB to $\beta$-Cys ${ }^{82}$. Interestingly, when we employed an in vivo assembled GtCPES:PEB complex (obtained by coexpressing GtCPES with PEB biosynthesis genes), we obtained a fluorescent product that resembled even better the native holo-PmCpeB with a fluorescence emission at $571 \mathrm{~nm}$ (data not shown).

Our negative control, $P m$ CpeB_C82A, displayed basal interaction with free 3(Z)-PEB visualized by an initial increase but subsequent decrease in fluorescence emission at $615 \mathrm{~nm}\left(\lambda_{\mathrm{ex}}=550 \mathrm{~nm}\right)$ (Figure 1C). Employing the PEB-loaded GtCPES lyase, no transfer was observed indicated by negligible fluorescence emission at $616 \mathrm{~nm}\left(\lambda_{\mathrm{ex}}=550 \mathrm{~nm}\right)$ (Figure 1D). As expected, no covalent attachment was observed in zinc-blot analysis (Figure 1E). Although we have previously shown that GtCPES can also bind the semi reduced intermediate 15,16-DHBV (14), DHBV-loaded lyase did not result in transfer of DHBV to PmCpeB (data not shown). As previously shown, DHBV is also not able to spontaneously attach to $P m \mathrm{CpeB}$ (17).

Two glutamate residues are important for PEB binding. Now that we established the assistance (chaperon) function of GtCPES during PEB transfer, we went ahead to perform a rigorous analysis to identify amino acid residues involved in substrate binding and transfer. Despite several approaches for co-crystallization of the lyase with its substrate PEB, no co-crystal structure was obtained. However, one molecule of 1,6-hexanediol was found within the barrel in the original structure giving a hint for the putative substrate binding site and allowing the initial identification of two residues involved in substrate binding (14). We now used the crystal structure and an amino acid sequence alignment of S-type lyases to identify ten individual amino acid residues potentially involved in PEB binding and transfer (Figure 2, Figure S1, (14)). These residues were exchanged to alanine residues using site directed mutagenesis. The following variants were generated: R18A, H21A, N22A, W69A, W75A, E136A, R146A, R148A, S150A and E168A (Figure 2). Residues E136, R146, and R148 are highly conserved among S-type lyases and are in close proximity to the 1,6-hexanediol molecule within the crystal structure of GtCPES and likely involved in substrate binding/coordination (14). Most of the other amino acid residues are located within the barrel and have potential influence on PEB binding. W75, which is located at the top of the lyase barrel, almost like a lid over the barrel, has putative influence on the interaction with the apo-protein $\mathrm{CpeB}$ (Figure 2). All variants were produced and purified in sufficient amounts and tested for their ability to bind PEB. In addition, thermal shift assays were performed to evaluate the effects the sequence variations on protein integrity and stability (21)

Upon binding to the wt GtCPES lyase, the absorbance maximum of PEB is red shifted from $535 \mathrm{~nm}$ to $598 \mathrm{~nm}$ with a significant increase in intensity with a shoulder at $557 \mathrm{~nm}$ (14). Five of the investigated GtCPES variants (R18A, N22A, W75A, R148A and S150A) showed almost identical binding spectra with a long wavelength major peak and a shoulder at a shorter wavelength (Table 1). These variants were considered wt-like. The remaining five variants displayed moderate to considerable changes from the wt GtCPES:PEB spectrum (Figure 3). The spectrum of variant W69A together with PEB still displayed two absorption maxima. However, what appears as a shoulder in the spectrum of the wt lyase, is a more distinct peak at $549 \mathrm{~nm}$ in this variant (Figure 3). The variants $\mathrm{H} 21 \mathrm{~A}$ and E168A also displayed two absorption peaks when incubated with PEB. However, there appears to be a large proportion of unbound PEB in these samples due to the absence of an increase in extinction coefficient of PEB upon incubation with the variants. In addition, the peak maxima were rather broad suggesting that the chromophore is less stretched, again suggesting a larger proportion of free PEB in the sample (22). In contrast, variants E136A and R146A did not bind PEB as a spectrum almost identical to that of free PEB was observed (Figure 3). The lack of PEB binding of the R146A variant is likely due to (partly) misfolded/aggregated protein. No discrete melting point between 47 and $58^{\circ} \mathrm{C}$ was obtained, as contrary to other mutants. The latter was also true for variant H21A. Therefore, both amino acid residues are likely important for structural integrity of the protein. Both variants were not investigated further. Interestingly, all variants that showed changes in their absorption properties with bound PEB, also 
displayed fluorescence emission at $\sim 638 \mathrm{~nm}$ when excited at $550 \mathrm{~nm}$ (Table 1). This is likely due to unspecific interaction of PEB with the lyase variants and differs from the wt protein where no fluorescence upon PEB binding was observed. In conclusion, both glutamate residues, E136 and E168 are strongly involved in PEB binding, while W69 has only minor influence on binding the substrate. $\mathrm{H} 21$ and R146 are important for structural integrity.

A tryptophan residue is important for PEB transfer to PmCpeB. We next tested whether the generated GtCPES variants that still showed PEB binding were also able to transfer PEB to apo-PmCpeB. Transfer of PEB was monitored using fluorescence spectroscopy as described above. Most of the variants that did show wt-like binding of the substrate PEB, did also display this behavior for the transfer reaction (Figure 4, S2). Interestingly, variant W69A that only showed minor absorption changes upon PEB binding (see paragraph before) did show abnormal transfer behavior that resembled that of a non-lyases mediated spontaneous reaction. Such reactions are indicative of initial increase but subsequent decrease of fluorescence emission at $565 \mathrm{~nm}$ with an additional fluorescence emission maxima observed at longer wavelength. Therefore, W69 likely is involved in PEB binding and its exchange leads to a large proportion of unbound PEB, which is then transferred in a spontaneous, unspecific reaction to PmCpeB. The same was observed for variant E168A that already showed only weak to negligible PEB binding. This variant already displayed fluorescence emission when incubated with PEB ( $E_{\max }=642 \mathrm{~nm}$ ) which decreased upon incubation with PmCpeB. Transfer is observed but based on the fluorescence emission, which increased and decreased, it appears that there is a large proportion of spontaneous transfer taking place. Variant S150A showed a normal PEB binding spectrum but reduced transfer indicative of a low fluorescence intensity increase upon incubation with $\mathrm{PmCpeB}$. The most important residue for PEB transfer is a tryptophan located at the upper rim of the barrel. Variant W75A, although still being able to bind PEB like the wt lyase, is not able to facilitate the transfer of PEB to PmCpeB. In order to test whether tight binding and therefore reduced release rates of PEB might cause this observation; binding affinities of PEB to wt GtCPES and the W75A variant were determined. Interestingly, GtCPES_W75A is able to bind PEB more than 10-fold tighter than the wt protein suggesting that this tight binding might prevent the release and transfer of PEB (Table 2).

A single methionine residue determines substrate specificity. GtCPES possesses a narrow binding pocket harboring amino acid residues with bulky side chains. As a consequence, GtCPES has a very high substrate specificity towards PEB. Nevertheless it is also able to bind the biosynthetic precursor 15,16-DHBV (14). Binding of the PEB-isomer phycocyanobilin (PCB) was not observed likely due to a rigid double bond between $\mathrm{C} 15-\mathrm{C} 16$ resulting in a missing flexibility of D-ring to fit into the binding pocket (Figure 5C). In order to characterize the molecular determinants of the substrate specificity, we compared both binding pocket and sequence of GtCPES with the PCB-specific S-type lyase CpcS from Thermosynechococcus elongatus ( $\mathrm{TeCpcS}$ ) (Figure 5A, S1). Based on a comparison of the two available crystal structures of $\mathrm{TeCpcS}$ and GtCPES, it became obvious that $\mathrm{TeCpcS}$ possesses a binding pocket with amino acid residues with less bulky side chains $(14,23)$ We therefore went ahead to mutagenize the binding pocket of GtCPES with the aim to widen it. Subsequently, the variant proteins were tested for their ability to bind PCB as well. We started to exchange amino acid residues around position 67 because these residues specifically constrict the binding pocket (Figures 2, 5). Overall, methionine 67 indeed seems to be highly specific for determining the substrate specificity. M67A and M67V variants of GtCPES were still able to bind PEB as indicated by binding spectra of PEB that resembled the wt situation (Table 3). In addition, we tested whether these variants were still able to transfer PEB to $\mathrm{PmCpeB}$. Both methionine variants and the I64A variant displayed specific transfer of 3(Z)-PEB (Figure 6). Interestingly, the M67A variant had the strongest affinity towards PEB of all measured GtCPES variants (Table 2) indicating that the affinity alone does not determine whether the chromophore is released from the binding pocket of the lyase or not.

Interestingly, both methionine variants were also able to bind PCB. Incubation with PCB resulted in a shift of the PCB absorption maximum to higher wavelengths $(639 \mathrm{~nm})$ together with an increase of extinction reflecting phycobilin binding (Figure 5). PCB binding furthermore also resulted in a fluorescent complex formation with an emission maximum at $662 \mathrm{~nm}$ (Table 3). As both methionine variants displayed the same spectroscopic behavior, all following experiments were only conducted employing the M67A variant.

GtCPES_M67A binds PCB with high affinity in vitro and in vivo. With respect to the gained PCB binding ability of the M67A variant, we next wanted to determine the affinity towards PCB. One way to do so is to investigate whether the CPES variant will form a complex with PCB when all necessary 
genes are coexpressed in E. coli $(14,24)$ Coexpression resulted in intensely colored cells for GtCPES_M67A with PEB as well as PCB (Figure 7). This complex remained stable through the affinity chromatography indicating strong binding of $\mathrm{PCB}$. The latter was confirmed by determining the binding affinities for 3(Z)-PEB and 3(E)-PCB. The calculated $\mathrm{K}_{\mathrm{D}}$ values showed strong binding with $0.044 \mu \mathrm{M}$ and $7 \mu \mathrm{M}$ for PEB and PCB, respectively (Table 2). Wild type GtCPES on the other hand only formed an in vivo complex with PEB but not PCB ((14) and Figure 7). During the subsequent purification process of GtCPES, the phycobilin remained bound to M67A as monitored by UV/vis-spectroscopy (Figure 7B). Absorption spectra of the elution fraction from affinity chromatography showed binding spectra with maxima similar to in vitro binding of 3(E)-PCB and 3(Z)-PEB by GtCPES_M67A (Table 3). Moreover, binding of PCB resulted in the formation of a fluorescent complex with GtCPES_M67A. This is in agreement with the complex formed in vivo (Table 3). Extinction coefficients for formed complexes with GtCPES_M67A were determined assuming that all PBP-lyase molecules were loaded with PCB or PEB. Resulting coefficients are $29.56 \mathrm{mM}^{-1} \mathrm{~cm}^{-1}$ (GtCPES_M67A:PEB) at $600 \mathrm{~nm}$ and $15.23 \mathrm{mM}^{-1} \mathrm{~cm}^{-1}$ (GtCPES_M67A:PCB) at $640 \mathrm{~nm}$ in sodium phosphate buffer. Additionally, in vivo formed GtCPES_M67A:PEB complex was also able to transfer loaded PEB to PmCpeB detected by fluorescence spectroscopy (data not shown). Here, emission maxima were similar to in vitro formed complex mediated transfer.

GtCPES_M67A transfers PCB to Cys ${ }^{82}$ of PmCpeB but not to CpcB. Subsequently, we were interested whether the GtCPES variant M67A exhibited the ability to mediate the transfer of 3(E)-PCB to Cys ${ }^{82}$ of $P m C p e B$. At first, spontaneous attachment of 3(E)-PCB was monitored by fluorescence spectroscopy (Figure 8A). Here, fluorescence spectra revealed slow but continuous emission increase over time with maximum wavelengths of $640 \mathrm{~nm}(P m C p e B), 642 \mathrm{~nm}$ (PmCpeB_C82A) corresponding to unspecific interaction of $\mathrm{PCB}$ with the apo-PBP. Lyase mediated product formation was detected after adding PBP subunit (PmCpeB, PmCpeB_C82A) to in vitro formed complex of GtCPES_M67A and 3(E)-PCB, as described before. All spectra differed from spontaneous attachment with the emission intensity of the first spectrum after 1 min reaction time representing the fluorescent complex between 3(E)-PCB and GtCPES_M67A with maximum around $663 \mathrm{~nm}$ (Figure 8D and Table 2). Over time, this emission maximum shifted to shorter maxima wavelengths $(651 \mathrm{~nm})$ indication transfer of PCB to the apoprotein. Similar fluorescence emission maxima $\left(E_{\max }=647 \mathrm{~nm}\right)$ were observed using $\mathrm{CpcS}$ lyase mediated PCB transfer to the $\mathrm{CpcB}$ subunit (Figure S2). The control experiment employing CpeB_C82A did not show any changes in absorption or fluorescence and only displayed the specific fluorescence background of GtCPES:PCB (Figure 8B,E). Covalent attachment of PCB to the apo-CpeB was further confirmed using zinc blot analysis (data not shown) (25). Unfortunately, attempts to detect chromopeptides using mass spectrometry failed.

In a final experiment, we asked the question whether the PCB-binding GtCPES_M67A variant would be able to transfer PCB to the phycocyanin $\beta$-subunit (CpcB) of Synechococcus sp. PCC7002. Unfortunately, here the results were not conclusive. The presence of the lyase led to an attachment product that showed fluorescence emission at $659 \mathrm{~nm}$, only $\sim 3-4 \mathrm{~nm}$ different to the spontaneous attachment (Figure 8C,F). However, the emission maximum also differed significantly from that mediated by the $\mathrm{CpcS}$ lyase, which resulted in a $\mathrm{CpcB}$ with a fluorescence emission at $647 \mathrm{~nm}$ (Figure S3). We conclude that GtCPES_M67A is not able to quantitatively transfer PCB to CpcB. Whether small amounts of $\mathrm{PCB}$ were correctly transferred to $\mathrm{CpcB}$ has to be determined.

\section{DISCUSSION}

We have previously initially characterized and crystallized the PBP lyase CPES from G. theta. PBP lyases are proteins that assist the correct attachment of light harvesting phycobilin pigments to conserved cysteine residues within a phycobiliprotein (15). Once all phycobilins are attached, the individual phycobiliproteins assemble into larger structures, either into phycobilisomes (cyanobacteria and rhodophytes) or into soluble heterodimers (cryptophytes). GtCPES is an S-type PBP lyase and was postulated to attach PEB to $\mathrm{Cys}^{82}$ of the $\beta$-subunit $\mathrm{CpeB}$. Although we were not able to show the transfer to the PBP subunit from $G$. theta, a transfer was observed using CpeB from the cyanobacterium Prochlorococcus marinus MED4. Both proteins share 55\% sequence homology. We therefore conclude that GtCPES is a highly specific lyase for the attachment of $3(\mathrm{Z})$-PEB also to $\beta$-Cys ${ }^{82}$ of $\mathrm{CpeB}$ of $G$. theta. This $\beta$-subunit has in total three PEB molecules bound: an additional one with a double linkage at $\mathrm{Cys}^{50,61}$ and one at $\mathrm{Cys}^{155}(6,7)$. Accordingly, it is reasonable that GtCPES is highly specific and is 
only able to transfer 3(Z)-PEB but not 15,16-DHBV, although binding of both bilins is observed. GtCPES shows a very narrow substrate specificity to the phycobilin it is transferring. This is mainly due to a very confined binding pocket, which is characterized by several bulky amino acid residues that restrict binding to bilins containing a C15-C16 double bond like PCB or phytochromobilin (РФB) (14). However, S-type lyases from certain cyanobacteria that only possess the phycobilin $\mathrm{PCB}$, i.e. CpcS, appear to have a broader substrate specificity. The $\mathrm{CpcS}$ lyase from Thermosynechococcus elongatus for instance is an universal lyase being able to bind and transfer PEB, PCВ and РФВ (23). Within this current study, we identified several amino acid residues that are important for phycobilin binding, transfer and substrate specificity. PEB binding is primarily mediated by two glutamate residues, E136 and E168. Both residues are facing the interior of the pocket and are likely involved in coordinating two of the pyrrole nitrogens of the bilin. E136 is highly conserved in the family of S-type lyases. W75 is also highly conserved within the S-type lyases and located at the upper rim of the barrel. Here, an involvement in the interaction with the apo-PBP is postulated. Interestingly, the GtCPES_W75A variant had a significantly higher binding affinity to the substrate. We therefore initially postulated that a high affinity retains the bilin within the lyase and therefore prevents the transfer to the apo-protein. However, this is in contrast to the data observed for the GtCPES M67A variant that displayed an even higher binding affinity towards PEB $(0.044 \mu \mathrm{M}$ for M67A vs. $0.86 \mu \mathrm{M}$ for W75A) implying that the affinity to the substrate does not determine whether the bilin is transferred or not. We rather postulate that a correct interaction with the target PBP must be present for a correct transfer to occur. This postulate is in conclusion with our data showing that the GtCPES_M67A variant is unable to transfer the bound $\mathrm{PCB}$ to the $\mathrm{CpcB}$ subunit, but only to the $\mathrm{CpeB}$ subunit. There might be smaller structural differences that determine the specific interaction of the CPES lyase with its corresponding (or in our case homolog) apo-protein.

Finally, we identified a crucial amino acid residue for substrate specificity. M67 determines the narrow substrate specificity of PEB-specific S-type lyases. This residue is highly conserved among CpeS lyases and is only substituted by an isoleucine in some cases (Figure S1). Within the PCB specific S-type lyases, this position is occupied by valine. With its shorter side chain, a valine residue at this position widens the pocket and allowing the more rigid PCB to enter the pocket. The same hold true for our M67A and M67V variants. When the long side chain of methionine is modified to either alanine or valine, the substrate specificity is broadened and the protein can bind both, PEB and PCB. We therefore hypothesize a crucial function for amino acid residue at position 67 in determining the substrate specificity of S-type lyases. Overall, our data provide very strong support for a dual function of the lyase both as bilin-selective binder and bilin-prepositioning chaperon for the hand-over to a dedicated target. 
Phycobiliprotein lyase substrate specificity

\section{EXPERIMENTAL PROCEDURES}

\section{Materials}

All chemicals were American Chemical Society grade or better unless specified otherwise. Expression vector pCOLADuet-1 was obtained from Novagen; pASK-IBA7+ was from IBA Life Sciences, pETDuet1 from Merck KgaA, and pGro7 was from TaKaRa. Strep-Tactin ${ }^{\circledR}-S e p h a r o s e$ from IBA, and TALON ${ }^{\circledR}$ metal affinity resin from Clontech were used. HPLC-grade acetone, acetonitrile, formic acid, and spectroanalytical grade glycerol were obtained from J.T. Baker. Sep-Pak cartridges were obtained from Waters.

\section{Construction of expression plasmids and site directed mutagenesis}

For construction of pCOLAcpeB a synthetic gene codon optimized for E. coli K12 (GENEius algorithm, MWG Eurofins Operon) encoding the $с p e B$ gene from Prochlorococcus marinus MED4 was PCR amplified with primers (Table 4) encompassing selected recognition sites (EcoRI, HindIII) for cloning into pCOLADuet (Novagen).

For construction of pCOLAcpcB the plasmid pBS150v_7002cpcB was used (26) This construct was a gift of W. M. Schluchter (University of New Orleans). The cpсB gene from Synechococcus sp. PCC7002 was PCR amplified (Table 4) with same recognition sites as for cpeB for following cloning into pColaDuet.

The construction of the plasmids pGtCPES, pE136A, pR146A, pR148A, pC149A, pETGtCPES, pTDho1pcyA and pTDho1pebS were described before $(14,24,27)$ All additional site-directed variants of GtCPES and PmCpeB were generated from pGtCPES or pCOLACpeB using the QuikChange ${ }^{\circledR}$ sitedirected mutagenesis kit (Stratagene) with help of primers listed in Table 4 (only the forward primer is shown, the reverse primer is the complement, codon changes are underlined). The resulting plasmids were verified by sequencing.

\section{Production and purification of recombinant proteins}

GtCPES and all variants as well as $\mathrm{CpcS}$ were produced and purified as described before $(14,28)$ $P m C p e B$ and $\mathrm{CpcB}$ were produced expressing pCOLAcpeB, pCOLAcpeB_C82A or pCOLAcpcB in E. coli BL21(DE3)-RIL. Cells were grown at $37^{\circ} \mathrm{C}$ in LB medium supplemented with $50 \mu \mathrm{g} / \mathrm{ml}$ kanamycin, and for среB with $100 \mathrm{mM}$ D-sorbitol and $2.5 \mathrm{mM}$ betaine to $\mathrm{OD}_{578 \mathrm{~nm}}$ of $0.5-0.6$. Subsequently, cells were induced by isopropyl- $\beta$-D-thiogalactopyranoside (IPTG; $0.5 \mathrm{mM}$ ) and incubated overnight at $17^{\circ} \mathrm{C}$. Cells were harvested by centrifugation, resuspended in lysis buffer (50 mM Tris- $\mathrm{HCl} \mathrm{pH} 7.5,100 \mathrm{mM} \mathrm{NaCl}, 10 \%$ Glycerol) and lysed by two or three passages through LM10 Microfluidizer ${ }^{\circledR}$ High Shear fluid homogenizer (Microfluidics) (cpeB, $c p c B$, gtCPES constructs) at $19.000 \mathrm{psi}$ or by three times 3 min sonification (50\%, KE76, Sonopuls HD6600, Bandelin) (only gtCPES constructs).

PmCpeB, its variant PmCpeB_C82A and $\mathrm{CpcB}$ were purified by affinity chromatography using TALON ${ }^{\circledR}$ metal affinity resin (Clontech) and purification was carried out according to the manufacturer's instructions based on sodium phosphate buffer $(60 \mathrm{mM}, 300 \mathrm{mM} \mathrm{NaCl}, \mathrm{pH}$ 7.5). For imidazole removing a dialysis against 100 fold volume of sodium phosphate buffer $(60 \mathrm{mM}$ sodium phosphate, $300 \mathrm{mM} \mathrm{NaCl}, \mathrm{pH} 7.5)$ over night at $4{ }^{\circ} \mathrm{C}(120 \mathrm{rpm})$ was performed.

\section{Determination of protein concentration}

Purified proteins were concentrated using Amicon ${ }^{\circledR}$ Ultra-4 Ultracel ${ }^{\circledR}-10 \mathrm{~K}$ (MWCO: $10.000 \mathrm{Da}$; Merck). Protein concentrations were quantified using the calculated molar extinction coefficient $\varepsilon_{280}$ (29).

\section{Coproduction of phycobilins and PBP-lyases}

Heterologous coproduction of GtCPES or variant GtCPES_M67A and PEB biosynthesis enzymes was performed in E. coli BL21(DE3) containing pET-constructs of gtcpeS and gtcpes_M67A (pET_gtCPES; pET_M67A) and pho1pebS or pTDho1pcyA. Cultures were grown in LB medium supplemented with $100 \mathrm{mM}$ sorbitol and $2.5 \mathrm{mM}$ betaine at $37^{\circ} \mathrm{C}, 100 \mathrm{rpm}$ to an $\mathrm{OD}_{578 \mathrm{~nm}}$ of 0.6 prior to induction with $0.1 \mathrm{mM}$ IPTG and incubated overnight at $17^{\circ} \mathrm{C}(\sim 16 \mathrm{~h})$. After cell harvesting by centrifugation, cells were resuspended in lysis buffer $(60 \mathrm{mM}$ sodium phosphate, $300 \mathrm{mM} \mathrm{NaCl}, \mathrm{pH} 7.5)$, and disrupted by pressure based homogenizer (LM10 Microfluidizer ${ }^{\circledR}$, Microfluidics), preferably, or sonification. Purification process was described before (14)

\section{Determination of extinction coefficient for PBP-lyase:phycobilin complex}

Extinction coefficients were determined for in vivo produced and purified PBP lyase:phycobilincomplexes. Here, $30 \mu \mathrm{M}$ of PEB or PCB were added to $20 \mu \mathrm{M}$ of GtCPES or variant GtCPES_M67A, 
Phycobiliprotein lyase substrate specificity

total volume was $550 \mu 1$. Phycobilin excess was removed by gel filtration (PD Minitrap G25 Sephadex column, GE healthcare) and performed according to the manufacturer's instructions with spin protocol based upon centrifugation (3000 rpm, 2 min). PBP-lyase concentration was determined by UV/visspectroscopy between all steps. Assuming that all PBP-lyase molecules were loaded with phycobilins, extinction coefficient for PBP-lyase: PEB complex at $600 \mathrm{~nm}$ and PBP-lyase:PCB complex at $640 \mathrm{~nm}$ were calculated with aid of Lambert-Beer law.

Determination of extinction coefficient for that complex $\left(\varepsilon_{600}=27.79 \mathrm{mM}^{-1} \mathrm{~cm}^{-1}\right)$ enabled the calculation of complex bound protein concentration under the assumption that all GtCPES molecules were present in complex with PEB.

\section{Thermal shift experiments}

The temperature of the unfolding transition midpoint, for excluding effect due to denaturation of GtCPES variants, was determined in thermal shift experiments $(30,31)$ For determination fluorescent protein stain SYPRO® Orange (5000x, Sigma-Aldrich) and CFX Connect Real-Time PCR Detection system (Bio-Rad) were used. Microplates were obtained from Biozym. With exclusion of light $50 \mu 1$ total volume composed of $5 \mu 1$ protein $\left(2 \mathrm{mg} \mathrm{ml}^{-1}\right), 5 \mu 1$ SYPRO® Orange (100x) and sodium phosphate buffer $(60 \mathrm{mM}$ sodium phosphate, $300 \mathrm{mM} \mathrm{NaCl}, \mathrm{pH} 7.5)$ were tested in triplicate. Controls were conducted without protein component. Melting temperatures were determined automatically by software CFX Maestro.

\section{Preparation of phycobilins}

PCB (3(E)-PCB) was isolated from Spirulina cells as described previously (32). Production, purification and preparation of PEB were described before (14) but PEB was concentrated by Advance Alpha 2-4 LSCplus freeze dryer (Christ). The vacuum was set to 0.04 mbar with ice condenser set at $-40^{\circ} \mathrm{C}$. Phycobilins were resuspended in an appropriate amount of DMSO before use. The isolated phycobilins were analyzed in terms of isomers via HPLC using Luna $5 \mu \mathrm{C} 18$ column (Phenomenex) and stored at $20{ }^{\circ} \mathrm{C}$ on silicagel orange in the dark. If isomer ratio showed strong excess of $E$ or $Z$-isomer for PEB or $\mathrm{PCB}$, concentrations were determined using $\varepsilon_{571}: 46.9 \mathrm{mM}^{-1} \mathrm{~cm}^{-1}$ (3(E)-PEB) in $\mathrm{MeOH} / 5 \% \mathrm{HCl}$ and $\varepsilon_{685}$ : $37.15 \mathrm{mM}^{-1} \mathrm{~cm}^{-1}(3(E)-\mathrm{PCB})$ in $\mathrm{MeOH} / 2.5 \% \mathrm{HCl}$ (33). Due to the absence of a reported extinction coefficient for 3(Z)-PEB $\varepsilon_{571}$ of the related 3(E)-PEB was used. 3(Z)-PEB was directly applied in experiments without separation of isomers and further purification because of $Z$-isomer excess.

\section{Phycobilin binding and transfer by GtCPES variants}

For binding studies excess of GtCPES or GtCPES variant $(20 \mu \mathrm{M})$, respectively, and phycobilin $(5 \mu \mathrm{M})$ were mixed in sodium phosphate buffer, $\mathrm{pH}$ 7.5. Absorption spectra (Agilent 8453 UV-visible spectrophotometer) and in case of transfer studies fluorescence emission spectra (Series 2, FA-256, Aminco Bowman) of the mixture and free phycobilins in the same buffer were detected. Measurements were done at $60 \%$ sensitivity and 975 V. For transfer studies $20 \mu \mathrm{M} \mathrm{PmCpeB}, P m \mathrm{CpeB} \_\mathrm{C} 82 \mathrm{~A}$ or CpcB was added 2 min after GtCPES (variant):phycobilin-complex formation and fluorescence emission was measured after 1, 5, 10, 15, 20, 30 and 45 min (Exc. $550 \mathrm{~nm}$ PEB; Exc. 600/620 nm PCB). All samples were incubated and measured at room temperature. After an incubation time of $45 \mathrm{~min}$ all sampled were prepared for and separated by SDS-PAGE on a $12.5 \%$ gel. Proteins were transferred to PVDF membrane that was subsequently incubated in $1.3 \mathrm{M}$ zinc acetate for $1 \mathrm{~h}$ at $4{ }^{\circ} \mathrm{C}$, and afterward zinc-enhanced fluorescence of proteins covalently associated with bilins was visualized under UV light (312 nm) (25)

\section{Spectroscopic analysis of phycobilin binding}

Increasing amounts of GtCPES (variant) $(2-15 \mu \mathrm{M})$ were added to $10 \mu \mathrm{M}$ (final concentration) 3(Z)PEB or 3(E)-PCB in a final volume of $200 \mu \mathrm{l}$ of sodium phosphate buffer, $\mathrm{pH} 7.5$, under exclusion from light. After incubation for $2 \mathrm{~min}$ absorbance spectra were recorded using Agilent $8453 \mathrm{UV}$-visible spectrophotometer. The method was described by Frankenberg and Lagarias (34) and was adapted, here. Analysis of spectra was performed using Microsoft Excel.

To obtain phycobiliprotein lyase dissociation constants, absorbance differences $(\Delta A)$ at the $\lambda_{\max }$ of each complex were plotted as a function of GtCPES concentration with aid of Microsoft Excel. Dissociation constants $K_{D}$ were obtained by fitting the parameters of the equation (1) to the experimental data, where $\left[\mathrm{P}_{\text {total }}\right]$ describes the total concentration of GtCPES, [ $\left.\mathrm{L}_{\text {total }}\right]$ of phycobilin and [PL] is the concentration of complex.

$$
K_{D}=\frac{\left(\left[P_{\text {total }}\right]-[P L]\right)\left(\left[L_{\text {total }}\right]-[P L]\right)}{[P L]}
$$


Phycobiliprotein lyase substrate specificity

\section{Data and image processing}

Spectra were generated in Origin ${ }^{\circledR}$ data analysis/graphic software, models of protein structure or ligand binding pocket were edited with aid of published GtCPES crystal structure (PDB code 4TQ2) by Pymol (35). Cavity model of GtCPES binding pocket was displayed by surface cavity mode of Pymol.

\section{ACKNOWLEDGEMENTS}

This work was supported by a grant from the Deutsche Forschungsgemeinschaft to NFD. We like to thanks Wendy Schluchter and Kai-Hong Zhao for the gift of the expression plasmids.

\section{CONFLICT OF INTEREST}

The authors declare that they have no conflicts of interest with the contents of this article.

\section{AUTHORS CONTRIBUTION}

NT, KEO, AP, EH and NFD designed the research, NT, KEO, MA performed the experiments, AP helped analyzing data, NT, KEO, NFD analyzed data and wrote the manuscript. All authors approved the manuscript. 
Phycobiliprotein lyase substrate specificity

\section{REFERENCES}

1. Glazer, A. N. (1985) Light harvesting by phycobilisomes. Annu Rev Biophys Biophys Chem 14, 47-77

2. Glazer, A. N., and Wedemayer, G. J. (1995) Cryptomonad biliproteins - an evolutionary perspective. Photosynth Res 46, 93-105

3. Spear-Bernstein, L., and Miller, K. R. (1989) Unique location of the phycobiliprotein lightharvesting pigment in the cryptophyceae. $J$ Phycol 25, 412-419

4. Wedemayer, G. J., Kidd, D. G., and Glazer, A. N. (1996) Cryptomonad biliproteins: Bilin types and locations. Photosynth Res 48, 163-170

5. Hoef-Emden, K. (2008) Molecular phylogeny of phycocyanin-containing cryptophytes: Evolution of biliproteins and geographical distribution. J Phycol 44, 985-993

6. Doust, A. B., Marai, C. N., Harrop, S. J., Wilk, K. E., Curmi, P. M., and Scholes, G. D. (2004) Developing a structure-function model for the cryptophyte phycoerythrin 545 using ultrahigh resolution crystallography and ultrafast laser spectroscopy. J Mol Biol 344, 135-153

7. Wilk, K. E., Harrop, S. J., Jankova, L., Edler, D., Keenan, G., Sharples, F., Hiller, R. G., and Curmi, P. M. (1999) Evolution of a light-harvesting protein by addition of new subunits and rearrangement of conserved elements: crystal structure of a cryptophyte phycoerythrin at 1.63A resolution. Proc Natl Acad Sci U S A 96, 8901-8906

8. Overkamp, K. E., Frankenberg-Dinkel, N. (2014) Phycobiliproteins - Biosynthesis, Assembly and Applications. in The Porphyrin Handbook (Ferreira, G., Kadish, K.M., Smith, K.M., Guilard, R. ed.), World Scientific Publishing Company. pp 187-226

9. Apt, K. E., Collier, J. L., and Grossman, A. R. (1995) Evolution of the phycobiliproteins. J Mol Biol 248, 79-96

10. Broughton, M. J., Howe, C. J., and Hiller, R. G. (2006) Distinctive organization of genes for light-harvesting proteins in the cryptophyte alga Rhodomonas. Gene 369, 72-79

11. Jenkins, J., Hiller, R. G., Speirs, J., and Godovac-Zimmermann, J. (1990) A genomic clone encoding a cryptophyte phycoerythrin alpha-subunit. Evidence for three alpha-subunits and an N-terminal membrane transit sequence. FEBS Lett 273, 191-194

12. Gould, S. B., Fan, E., Hempel, F., Maier, U. G., and Klosgen, R. B. (2007) Translocation of a phycoerythrin alpha subunit across five biological membranes. J Biol Chem 282, 30295-30302

13. Frankenberg, N., Mukougawa, K., Kohchi, T., and Lagarias, J. C. (2001) Functional genomic analysis of the HY2 family of ferredoxin-dependent bilin reductases from oxygenic photosynthetic organisms. Plant Cell 13, 965-978

14. Overkamp, K. E., Gasper, R., Kock, K., Herrmann, C., Hofmann, E., and Frankenberg-Dinkel, N. (2014) Insights into the biosynthesis and assembly of cryptophycean phycobiliproteins. $J$ Biol Chem 289, 26691-26707

15. Scheer, H., and Zhao, K. H. (2008) Biliprotein maturation: the chromophore attachment. Mol Microbiol 68, 263-276

16. Bolte, K., Kawach, O., Prechtl, J., Gruenheit, N., Nyalwidhe, J., and Maier, U. G. (2008) Complementation of a phycocyanin-bilin lyase from Synechocystis sp. PCC 6803 with a nucleomorph-encoded open reading frame from the cryptophyte Guillardia theta. BMC Plant Biol 8, 56

17. Wiethaus, J., Busch, A. W., Kock, K., Leichert, L. I., Herrmann, C., and Frankenberg-Dinkel, N. (2010) CpeS is a lyase specific for attachment of 3Z-PEB to Cys82 of beta-phycoerythrin from Prochlorococcus marinus MED4. J Biol Chem 285, 37561-37569

18. Zhao, K. H., Zhang, J., Tu, J. M., Bohm, S., Ploscher, M., Eichacker, L., Bubenzer, C., Scheer, H., Wang, X., and Zhou, M. (2007) Lyase activities of CpcS- and CpcT-like proteins from Nostoc PCC7120 and sequential reconstitution of binding sites of phycoerythrocyanin and phycocyanin beta-subunits. J Biol Chem 282, 34093-34103

19. Steglich, C., Frankenberg-Dinkel, N., Penno, S., and Hess, W. R. (2005) A green lightabsorbing phycoerythrin is present in the high-light-adapted marine cyanobacterium Prochlorococcus sp. MED4. Environ Microbiol 7, 1611-1618 
20. Steglich, C., Post, A. F., and Hess, W. R. (2003) Analysis of natural populations of Prochlorococcus spp. in the northern Red Sea using phycoerythrin gene sequences. Environ Microbiol 5, 681-690

21. Lavinder, J. J., Hari, S. B., Sullivan, B. J., and Magliery, T. J. (2009) High-Throughput Thermal Scanning: A General, Rapid Dye-Binding Thermal Shift Screen for Protein Engineering. J Am Chem Soc 131, 3794-3795

22. Krois, D., and Lehner, H. (1993) Helically fixed chiral bilirubins and biliverdins: a new insight into the conformational, associative and dynamic features of linear tetrapyrrols. $J$ Chem SocPerkTransac 2 7, 1351-1360

23. Kronfel, C. M., Kuzin, A. P., Forouhar, F., Biswas, A., Su, M., Lew, S., Seetharaman, J., Xiao, R., Everett, J. K., Ma, L. C., Acton, T. B., Montelione, G. T., Hunt, J. F., Paul, C. E., Dragomani, T. M., Boutaghou, M. N., Cole, R. B., Riml, C., Alvey, R. M., Bryant, D. A., and Schluchter, W. M. (2013) Structural and biochemical characterization of the bilin lyase CpcS from Thermosynechococcus elongatus. Biochemistry 52, 8663-8676

24. Dammeyer, T., Bagby, S. C., Sullivan, M. B., Chisholm, S. W., and Frankenberg-Dinkel, N. (2008) Efficient phage-mediated pigment biosynthesis in oceanic cyanobacteria. Curr Biol 18, $442-448$

25. Berkelman, T. R., and Lagarias, J. C. (1986) Visualization of bilin-linked peptides and proteins in polyacrylamide gels. Anal Biochem 156, 194-201

26. Shen, G., Saunee, N. A., Williams, S. R., Gallo, E. F., Schluchter, W. M., and Bryant, D. A. (2006) Identification and characterization of a new class of bilin lyase: the cpcT gene encodes a bilin lyase responsible for attachment of phycocyanobilin to Cys-153 on the beta-subunit of phycocyanin in Synechococcus sp. PCC 7002. J Biol Chem 281, 17768-17778

27. Dammeyer, T., and Frankenberg-Dinkel, N. (2006) Insights into phycoerythrobilin biosynthesis point toward metabolic channeling. J Biol Chem 281, 27081-27089

28. Zhao, K. H., Su, P., Tu, J. M., Wang, X., Liu, H., Plöscher, M., Eichacker, L., Yang, B., Zhou, M., and Scheer, H. (2007) Phycobilin: cystein-84 biliprotein lyase, a near-universal lyase for cysteine-84-binding sites in cyanobacterial phycobiliproteins. Proc Natl Acad Sci USA 104, 14300-14305

29. Gill, S. C., and Von Hippel, P. H. (1989) Calculation of protein extinction coefficients from amino acid sequence data. Analytical biochemistry 182, 319-326

30. Boivin, S., Kozak, S., and Meijers, R. (2013) Optimization of protein purification and characterization using Thermofluor screens. Protein Expr Purif 91, 192-206

31. Niesen, F. H., Berglund, H., and Vedadi, M. (2007) The use of differential scanning fluorimetry to detect ligand interactions that promote protein stability. Nat Protoc 2, 2212-2221

32. Terry, M. (2002) Biosynthesis and Analysis of Bilins. in Heme, Chlorophyll, and Bilins (Smith, A., and Witty, M. eds.), Humana Press. pp 273-291

33. Weller, J. P., and Gossauer, A. (1980) Synthesen von Gallenfarbstoffen, X. Synthese und Photoisomerisierung des racem. Phytochromobilin-dimethylesters. Chem Ber 113, 1603-1611

34. Frankenberg, N., and Lagarias, J. C. (2003) Phycocyanobilin:ferredoxin oxidoreductase of Anabaena sp. PCC 7120. Biochemical and spectroscopic characterization. J Biol Chem 278, 9219-9226

35. DeLano, W. L. (2002) PyMOL. DeLano Scientific, San Carlos, CA 700 
Phycobiliprotein lyase substrate specificity

\section{TABLES}

TABLE 1. Binding and transfer of 3(Z)-PEB after incubation with different GtCPES variants compared to the wild type.

Melting points (in ${ }^{\circ} \mathrm{C}$ ) of proteins determined in triplicate and absorbance/emission maxima of binding/transfer are given. Complex $\mathrm{E}_{\max }$ : detected emission maximum after adding PBP-lyase to free PEB. $\mathrm{E}_{\max }$ : emission maximum after 45 min incubation time of GtCPES (variant):bilin with PmCpeB.Excitation wavelength was $550 \mathrm{~nm}$.

\begin{tabular}{lcccccc}
\hline $\begin{array}{l}\text { GtCPES } \\
\text { (variant) }\end{array}$ & $\mathbf{T}_{\mathbf{m}}\left[{ }^{\circ} \mathbf{C}\right]$ & $\begin{array}{c}\text { Binding } \\
\text { PEB }\end{array}$ & $\begin{array}{c}\mathbf{A}_{\max } \\
{[\mathbf{n m}]}\end{array}$ & $\begin{array}{c}\text { Complex } \\
\mathbf{E}_{\mathbf{m a x}}[\mathbf{n m}]\end{array}$ & $\begin{array}{c}\text { Transfer } \\
\mathbf{P E B}\end{array}$ & $\begin{array}{c}\mathbf{E}_{\max } \\
{[\mathbf{n m}]}\end{array}$ \\
\hline WT & $56.0 \pm 0.0$ & yes & 554,600 & --- & yes & 568 \\
& & & & & & \\
R18A & $48.0 \pm 0.0$ & yes & 552,593 & --- & $(\text { yes })^{\mathrm{a}}$ & 567 \\
N22A & $55.0 \pm 0.0$ & yes & 555,598 & --- & yes $^{\mathrm{a}}$ & 568 \\
W69A & $48.0 \pm 0.0$ & yes & 549,596 & 637 & no & 565 \\
W75A & $58.0 \pm 0.0$ & yes & 554,599 & --- & no & n.d. \\
E136A & $47.5 \pm 0.0$ & no & 539 & 638 & no & 565 \\
R148A & $56.0 \pm 0.0$ & yes & 552,600 & -- & yes & 566 \\
S150A & $48.2 \pm 0.3$ & yes & 554,600 & --- & $(\text { yes })^{\mathrm{a}}$ & 568 \\
& $57.5 \pm 0.0$ & & & & & \\
E168A & $49.0 \pm 0.0$ & (no) $^{\mathrm{b}}$ & 541,597 & 642 & $($ no) & 568 \\
\hline
\end{tabular}

$a$ Less increase of fluorescence emission compared to wtGtCPES. $b$ Binding spectra differs from wtGtCPES:PEB spectra. n.d., not detectable

\section{TABLE 2. Binding affinities for complex formation with PEB and PCB.}

Phycobilin binding analysis was performed as described were tested by titration described in the experimental procedures. $\mathrm{K}_{\mathrm{D}}$ values were calculated from equation (1) by using solver function of Microsoft Excel. Results were averaged over two measurements. n.b., no binding.

\begin{tabular}{lcc}
\hline $\begin{array}{l}\text { GtCPES } \\
\text { (variant) }\end{array}$ & $\begin{array}{c}\text { PEB } \\
\mathbf{K}_{\mathbf{D}}[\boldsymbol{\mu M}]\end{array}$ & $\begin{array}{c}\text { PCB } \\
\mathbf{K}_{\mathbf{D}}[\boldsymbol{\mu M}]\end{array}$ \\
\hline WT & $14.2 \pm 2.6$ & n.b. \\
M67A & $0.044 \pm 0.003$ & $7.0 \pm 0.4$ \\
W75A & $0.86 \pm 0.06$ & --- \\
\hline
\end{tabular}


bioRxiv preprint doi: https://doi.org/10.1101/2020.03.31.018853; this version posted April 1, 2020. The copyright holder for this preprint (which was not certified by peer review) is the author/funder, who has granted bioRxiv a license to display the preprint in perpetuity. It is made available under aCC-BY-NC 4.0 International license.

Phycobiliprotein lyase substrate specificity

TABLE 3. In vitro binding and transfer to PmCpeB of PEB and PCB after incubation with different GtCPES variants.

Melting points (in ${ }^{\circ} \mathrm{C}$ ) of proteins determined in triplicate and absorption/emission maxima of binding/transfer are presented. (Exc. $550 \mathrm{~nm}$ PEB; Exc. 600/620 nm PCB)

\begin{tabular}{|c|c|c|c|c|c|c|c|c|c|c|c|}
\hline \multirow{2}{*}{$\begin{array}{l}\text { GtCPES } \\
\text { (variant) }\end{array}$} & \multirow{2}{*}{$\begin{array}{c}\mathbf{T}_{\mathbf{m}} \\
{\left[{ }^{\circ} \mathbf{C}\right]}\end{array}$} & \multicolumn{5}{|c|}{ 3(Z)-PEB } & \multicolumn{5}{|c|}{$3(E)-P C B$} \\
\hline & & Binding & $\begin{array}{l}\mathbf{A}_{\max } \\
{[\mathrm{nm}]}\end{array}$ & $\begin{array}{l}\mathbf{E}_{\max } \\
{[\mathbf{n m}]}\end{array}$ & Transfer & $\begin{array}{l}\mathbf{E}_{\max } \\
{[\mathrm{nm}]}\end{array}$ & Binding & $\begin{array}{l}A_{\max } \\
{[\mathbf{n m}]}\end{array}$ & $\begin{array}{l}\mathbf{E}_{\max } \\
{[\mathbf{n m}]}\end{array}$ & Transfer & $\begin{array}{l}\mathbf{E}_{\max } \\
{[\mathbf{n m}]}\end{array}$ \\
\hline I65A & $\begin{array}{c}47.5 \pm \\
0.5\end{array}$ & yes & $\begin{array}{l}555 \\
599\end{array}$ & --- & yes & 568 & no & 616 & --- & no & 640 \\
\hline M67A & $\begin{array}{c}53.3 \pm \\
0.3\end{array}$ & yes & $\begin{array}{l}\mathbf{5 5 3} \\
\mathbf{5 9 8}\end{array}$ & $635^{b}$ & yes & 569 & yes & 639 & 662 & yes & 651 \\
\hline M67V & $\begin{array}{c}52.3 \pm \\
0.4\end{array}$ & yes & $\begin{array}{l}\mathbf{5 5 5} \\
\mathbf{5 9 7}\end{array}$ & --- & yes & 567 & yes & 638 & 663 & no & 640 \\
\hline
\end{tabular}

$a$, Absorbance spectrum differs from wtGtCPES:bilin concerning extinction. $b$, fluorescence only detected for in vivo binding. ND means not determined, n.d., not detectable.

TABLE 4. Constructed expression plasmids. Forward primers for site-directed mutagenesis are shown, reverse primer is the complement. Changed codons are underlined.

\begin{tabular}{|c|c|c|c|}
\hline Plasmid & Host vector & $\begin{array}{l}\text { Recognition } \\
\text { sites }\end{array}$ & Primers (5' - 3') \\
\hline \multirow[t]{2}{*}{ pCOLAcpeB } & pCOLADuet-1 & EcoRI & GCGAATTCTATGACAGTTTCAAAGAGTAAT \\
\hline & & HindIII & GCAAGCTTCTATCTTAAAAGATTAATTATT \\
\hline \multirow{2}{*}{ pCOLAcpcB } & pCOLADuet & EcoRI & CGGAATTCTATGTTTGATATTTTTACC \\
\hline & & HindIII & GCAAGCTTTTATTCAACAGCTTTTG \\
\hline pR18A & $\mathrm{p} G t C P E S$ & & GTTCTTGGCGCTCATTGGCTTCTAGCCACAATATTGC \\
\hline pH22A & $\mathrm{p} G t C P E S$ & & GCTCATTGCGTTCTAGCGCCAATATTGCCTTTGCGC \\
\hline pN23A & $\mathrm{p} G t C P E S$ & & GCGTTCTAGCCACGCTATTGCCTTTGCGCAACTGG \\
\hline pI65A & $\mathrm{p} G t C P E S$ & & GAAAAGGCCTGTTCAAGCGCTCGCATGAGTTGGGAAG \\
\hline pM67A & $\mathrm{p} G t C P E S$ & & GTTCAAGCATTCGCGCGAGTTGGG AAGGTAG \\
\hline pM67V & $\mathrm{p} G t C P E S$ & & CTGTTCAAGCATTCGCGTAAGTTGGGAAGGTAGCTC \\
\hline pW69A & $\mathrm{p} G t C P E S$ & & GCATTCGCATGAGTGCGGAAGGTAGCTCTGACTGG \\
\hline pW75A & $\mathrm{p} G t C P E S$ & & GGGAAGGTAGCTCTGACGCGGATGAAAACGAAGTG \\
\hline pE136D & $\mathrm{p} G t C P E S$ & & CTGCAGCCGAAGACCGCATTTGGTTTG \\
\hline pS150A & pGtCPES & & GATTTACGCATGCGTTGCGCCATCATCAAGACACAGC \\
\hline pE168A & $\mathrm{p} G t C P E S$ & & GCGAGTCTTTCGACCGCAGTACGCGACAAAAGC \\
\hline pI65A_M67A & $\mathrm{p} G t C P E S$ & & GGCCTGTTCAAGCECTCGCGCGAGTTGGGAAGGTAGC \\
\hline pI65A_M67V & pGtCPES & & 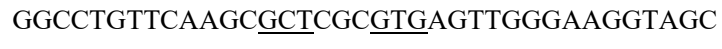 \\
\hline pI65A_M67V_L89P & pI65A_M67V & & GGCTCCACTGTCCTGGTTCCGTACAAAGATGAGG \\
\hline $\begin{array}{l}\text { pI65A_M67V_L89P_ } \\
\text { F123L }\end{array}$ & pI65A_M67V_L89P & & GCAGGAAGATGGGACCCTTGTGCTGCATACC \\
\hline pET_M67A & pET-GtCPES & & GTTCAAGCATTCGCGCGAGTTGGGAAGGTAG \\
\hline
\end{tabular}


Phycobiliprotein lyase substrate specificity

\section{FIGURES}

FIGURE 1.

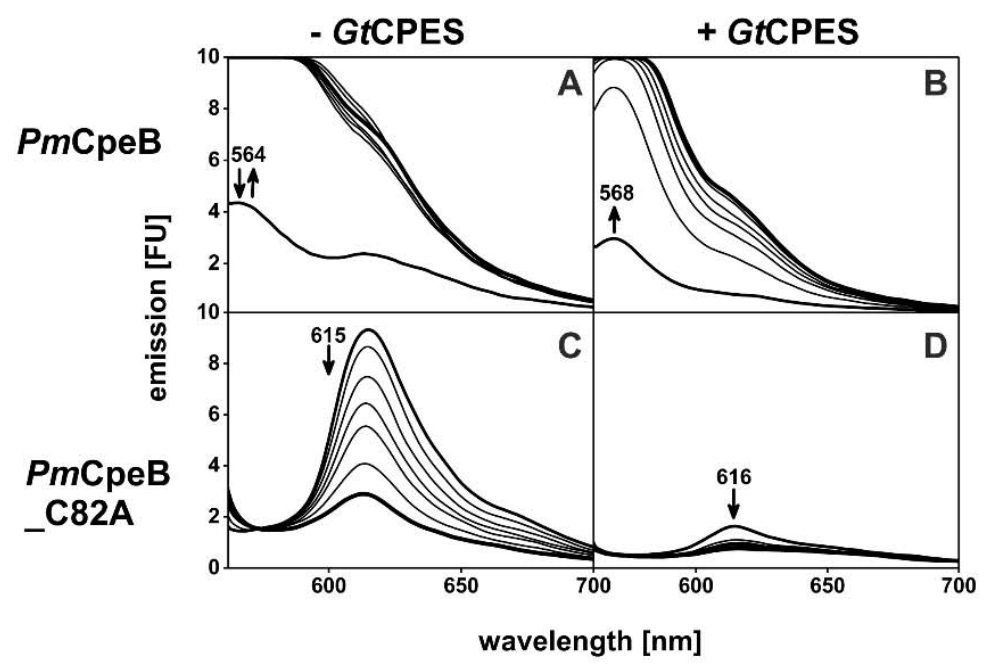

E

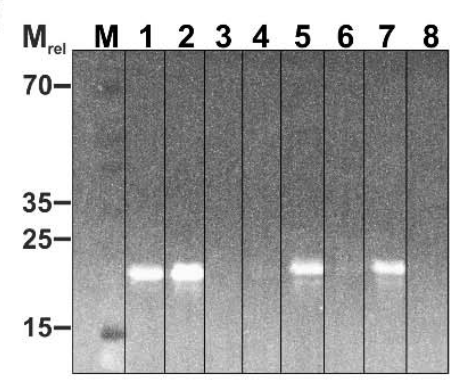

M: marker

1: wtGtCPES:PEB with PmCpeB

2: $\mathrm{PEB}$ with $P m \mathrm{CpeB}$

3: wtGtCPES:PEB with PmCpeB_C82A

4: PEB with PmCpeB_C82A

5: PCB with $P m$ CpeB

6: PCB with PmCpeB_C82A

7: GtCPES_M67A:PCB with PmCpeB

8: GtCPES_M67A:PCB with PmCpeB_C82A

FIGURE 1. GtCPES mediated and spontaneous PEB chromophorylation of PmCpeB or PmCpeB_C82A and covalent binding formation of PmCpeB with PCB or PEB visualized by zincenhanced fluorescence. The apo-PBPs PmCpeB or PmCpeB_C82A were incubated with PEB (A, C) or wtGtCPES:PEB complexes (B, D). Emission spectra $\left(\lambda_{\text {ex }}=550 \mathrm{~nm}\right)$ of 1, 5, 10, 15, 20, 30, and 45 min after addition of PmCpeB or PmCpeB_C82A, respectively, were detected. First and last spectra are shown by bold lines. Emission maxima are given and the course of fluorescence emission changes is indicated by arrows. After spontaneous and PBP-lyase mediated transfer studies of PEB and PCB, samples were used for SDS-PAGE and blotted onto PVDF membrane (E). Membrane was incubated for $1 \mathrm{~h}$ at $4{ }^{\circ} \mathrm{C}$ in $1.3 \mathrm{M}$ zinc acetate and detected under UV-light $(312 \mathrm{~nm})$. Fluorescence signals based on zinc-phycobilin complexation describe covalent binding of phycobilins to PmCpeB (with his-tag: $21.4 \mathrm{kDa}$ ). Absent signals indicate missing binding. 
Phycobiliprotein lyase substrate specificity

\section{FIGURE 2.}

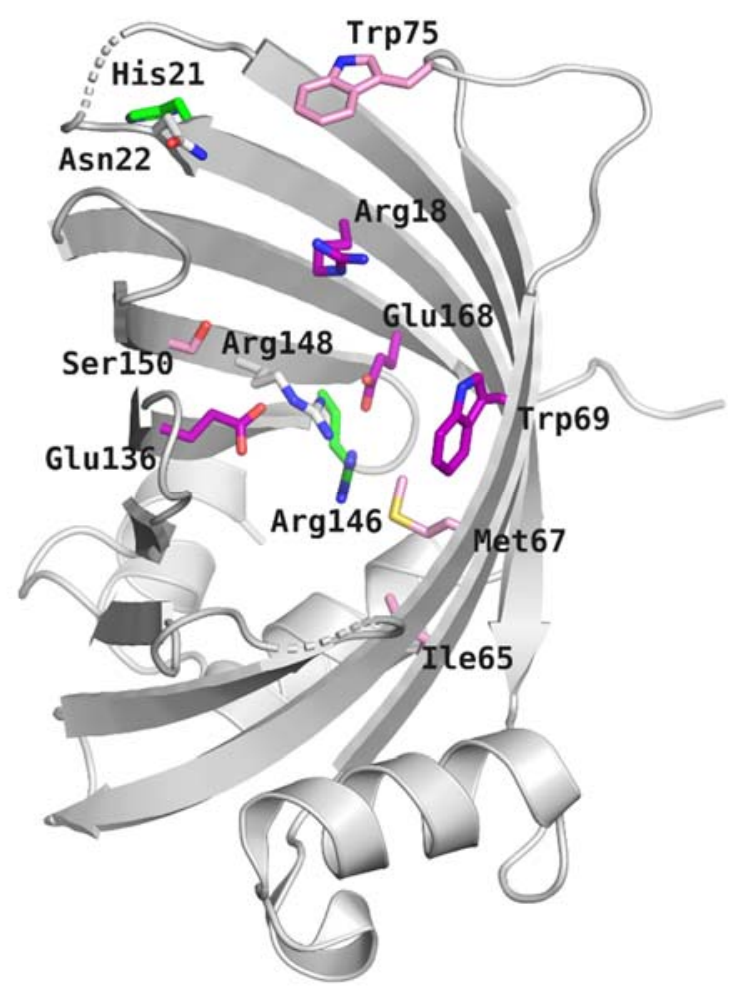

FIGURE 2. Location of amino acid residues of GtCPES investigated within this study. The structure of GtCPES (PDB code 4TQ2) is shown in cartoon representation. Residues discussed in the main text are shown as sticks with color coding representing the effect of a site specific exchange on GtCPES function: white - no change; green - structural defect; purple - bilin binding; pink - bilin transfer and selectivity. Figure generated with Pymol (35). 
Phycobiliprotein lyase substrate specificity

\section{FIGURE 3.}

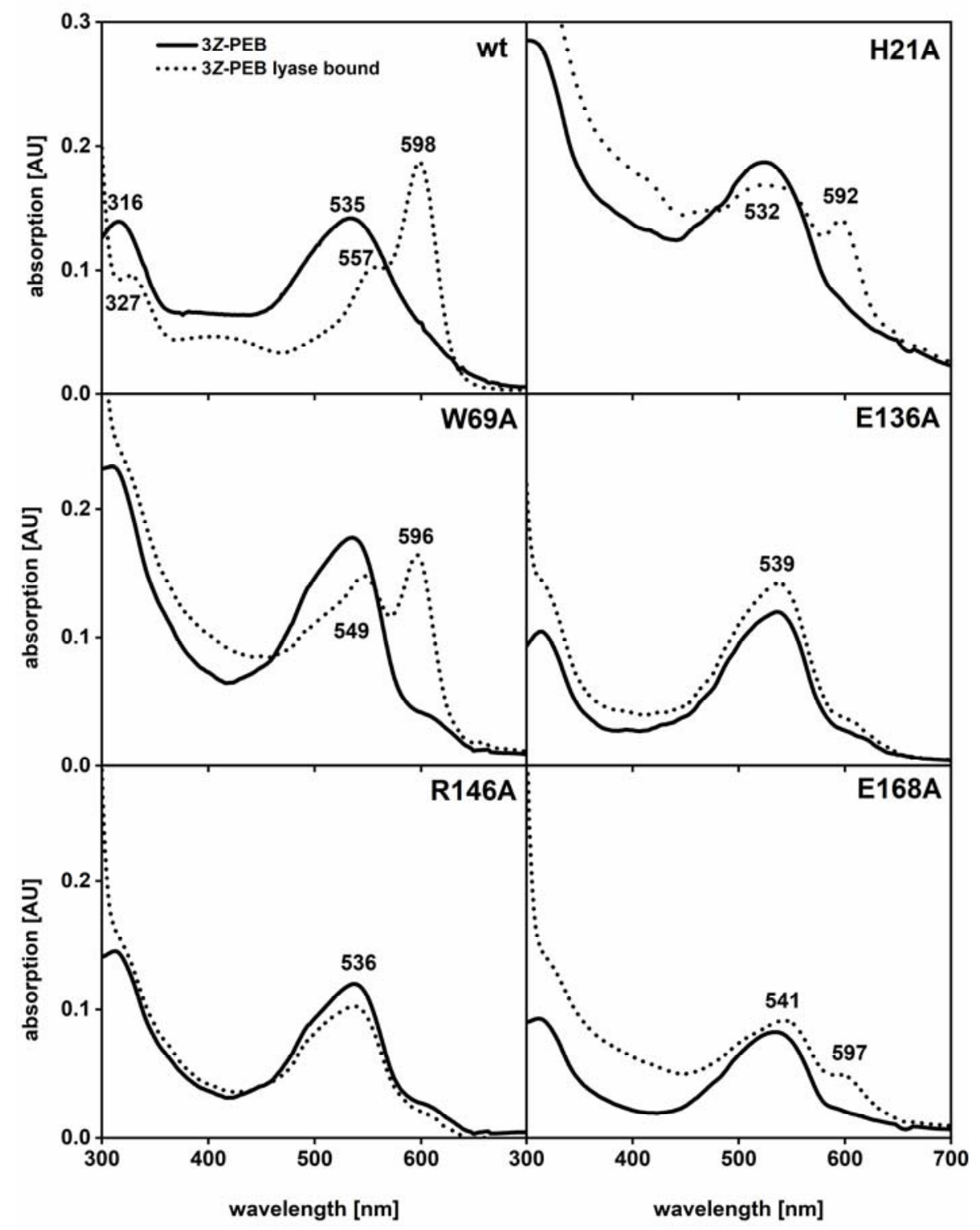

FIGURE 3. UV-Vis spectra of 3(Z)-PEB bound to GtCPES and variants. Free PEB spectra are shown as solid lines, the spectra of lyase variants with PEB are shown as dotted lines. Peak maxima are given and the respective amino acid exchange. 


\section{FIGURE 4.}

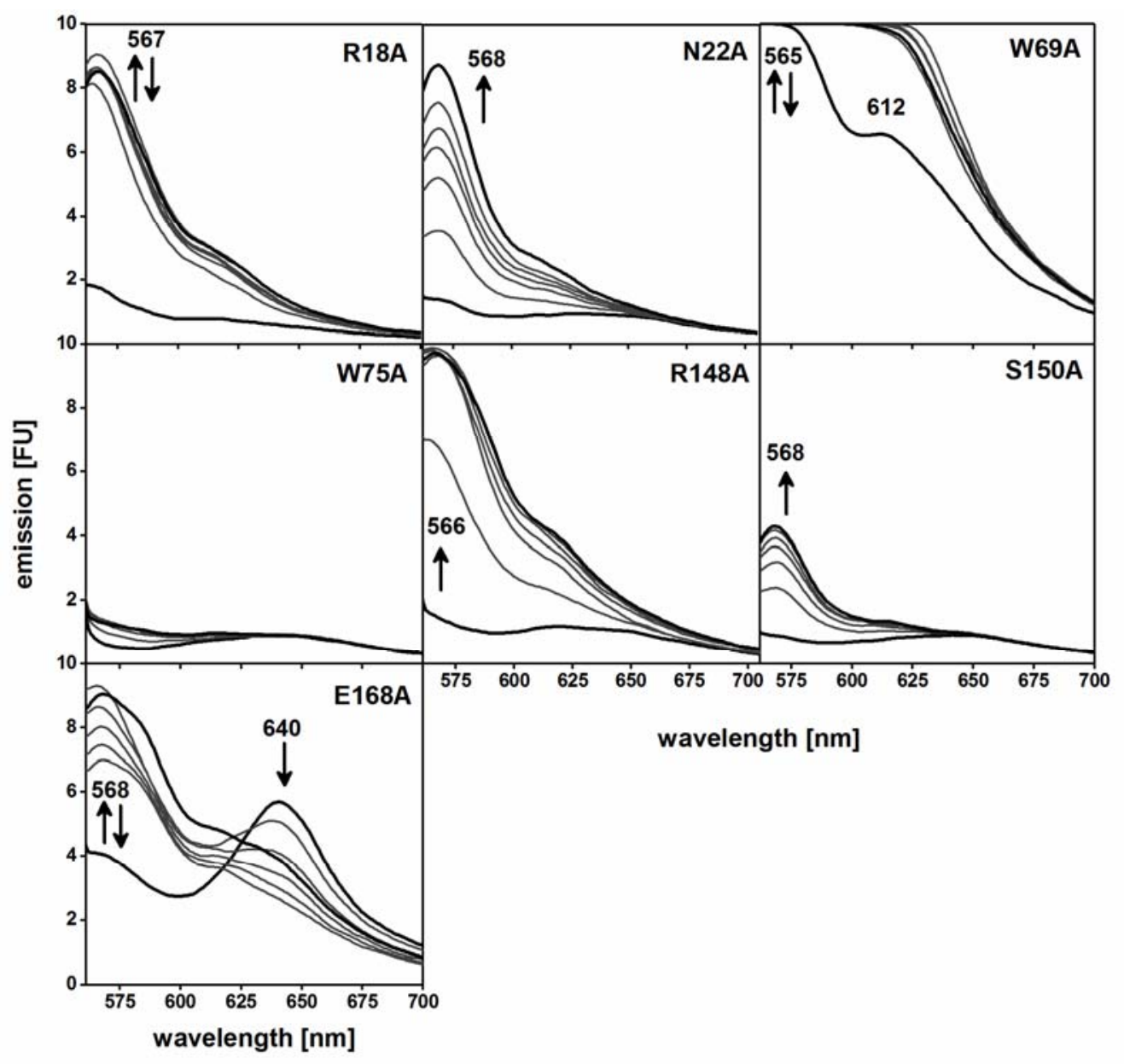

FIGURE 4. Transfer of 3(Z)-PEB to PmCpeB by GtCPES variants. Apo-PmCpeB was incubated with $G t C P E S$ variants preincubated with PEB and. Emission spectra $\left(\lambda_{\mathrm{ex}}=550 \mathrm{~nm}\right)$ at $1,5,10,15,20$, 30, and 45 min after addition of $P m C p e B$ were taken. First and last spectra are shown by bold lines. Emission maxima are given and the course of fluorescence emission changes is indicated by arrows. 
Phycobiliprotein lyase substrate specificity

\section{FIGURE 5.}

A

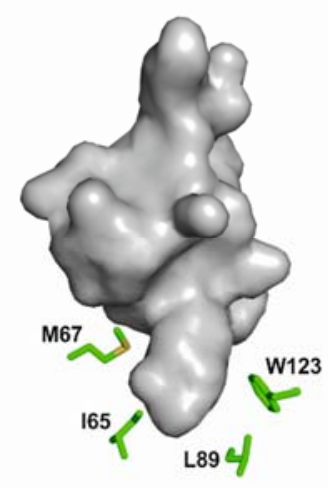

B

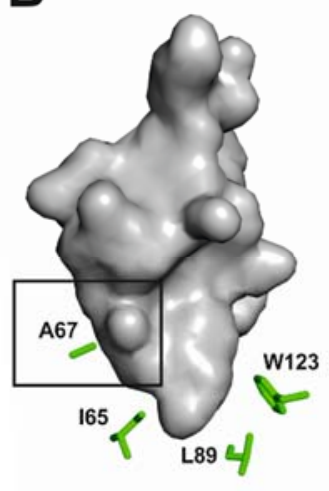

C

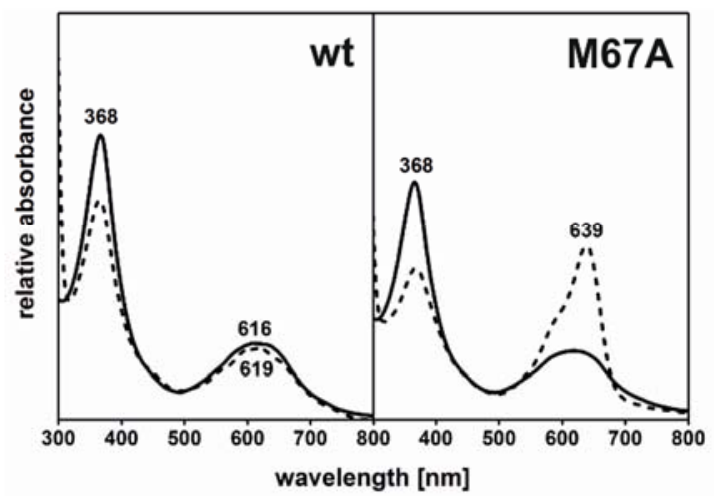

FIGURE 5. Transformation of PEB specific lyase into universal lyase. Comparison of a cavity model of the phycobilin binding pocket of GtCPES (A) and GtCPES_M67A (B). Model was generated using Pymol with aid of surface cavity mode (35). C. Absorption spectra of free 3(E)-PCB (solid line) compared with absorption spectra after addition of wt GtCPES (left panel) and GtCPES_M67A (right panel) (dashed line). Absorbance maxima are indicated. 


\section{FIGURE 6.}

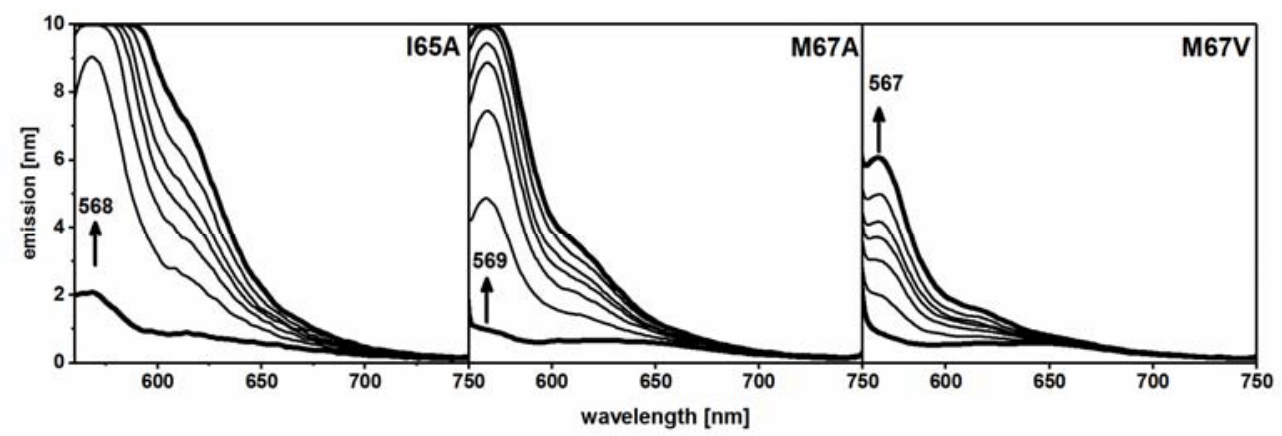

FIGURE 6. Transfer of 3(Z)-PEB to PmCpeB by GtCPES variants with larger binding pocket. Apo-PmCpeB was incubated with GtCPES variants preincubated with PEB. Emission spectra $\left(\lambda_{\mathrm{ex}}=550 \mathrm{~nm}\right)$ at $1,5,10,15,20,30$, and $45 \mathrm{~min}$ after addition of $P m C p e B$ were taken. First and last spectra are shown by bold lines. Emission maxima are given and the course of fluorescence emission changes is indicated by arrows. 
FIGURE 7.

\section{A}

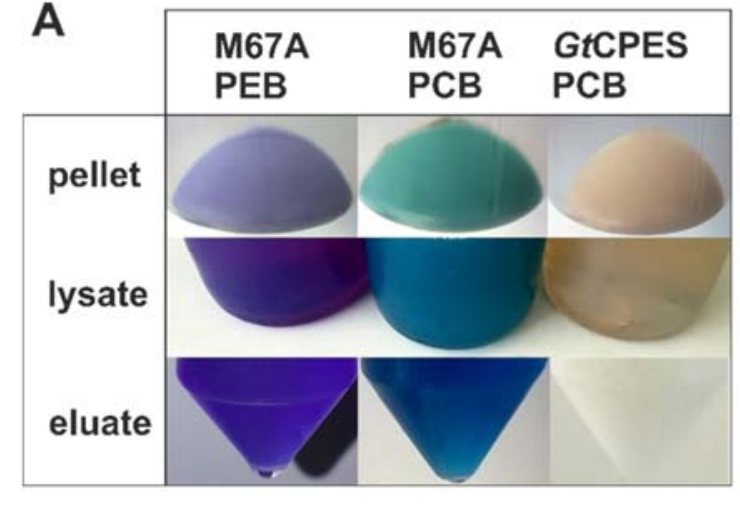

B

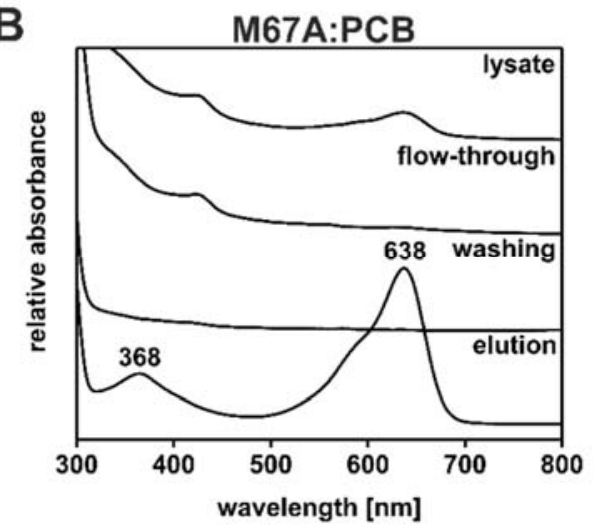

FIGURE 7. In vivo complex formation of GtCPES_M67A with PCB and PEB. Coproduction of wtGtCPES and variant GtCPES M67A with synthesis enzymes for PCB (Ho1, PcyA) and PEB (Ho1, PebS) were conducted in E. coli BL21(DE3). Formed GtCPES_M67A:bilin complexes were purified by affinity chromatography and detected by color formation (A) and UV/vis-spectroscopy (B; GtCPES_M67A:PCB). Absorption maxima in elution fraction are labelled. Coproduction of wtGtCPES, Hol and PcyA shows no color (control). 
FIGURE 8

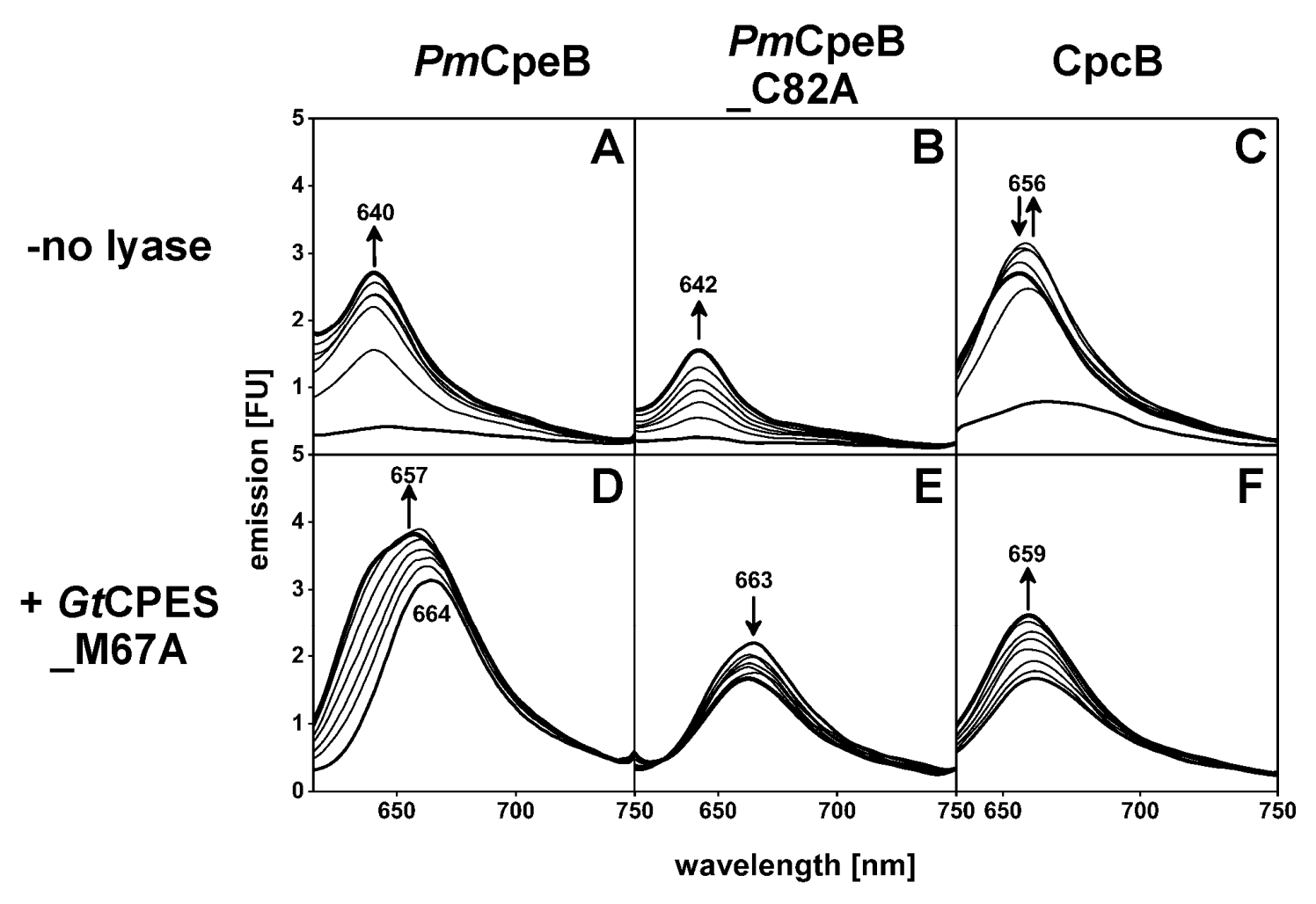

FIGURE 8. Spontaneous and GtCPES_M67A mediated PCB chromophorylation of PmCpeB, PmCpeB_C82A and CpcB The apo-PBPs PmCpeB, PmCpeB_C82A and CpcB were incubated with PCB (- no lyase) or GtCPES_M67A:PCB complexes. Emission spectra $\left(\lambda_{\text {ex }}=600 \mathrm{~nm}(\mathbf{A}, \mathbf{B}, \mathbf{D}, \mathbf{E})\right.$; $\lambda_{\text {ex }}=620 \mathrm{~nm}(\mathbf{C}$ and $\left.\mathbf{F})\right)$ of $1, \overline{5}, 10,15,20,30$, and $45 \mathrm{~min}$ after addition of apo-PBPs were detected. First and last spectra are shown by bold lines. Emission maxima are given and the course of fluorescence emission changes is indicated by arrows. 